\title{
The effect of melatonin on reduction in the need for sedative agents and duration of mechanical ventilation in traumatic intracranial hemorrhage patients: a randomized controlled trial
}

\author{
Farhad Soltani $^{1}$ - Amir Salari ${ }^{1}$. Fatemeh Javaherforooshzadeh ${ }^{1}\left[\right.$ D Nozar Nassajjian ${ }^{1} \cdot$ Farahmand Kalantari $^{1}$
}

Received: 17 March 2020 / Accepted: 16 July 2020 / Published online: 22 July 2020

○) Springer-Verlag GmbH Germany, part of Springer Nature 2020

\begin{abstract}
Purpose This study aimed to determine the effect of exogenous melatonin on the number of sedative drugs and the duration of mechanical ventilation in traumatic intracranial hemorrhage patients in ICU.

Methods This double-blind randomized clinical trial study was conducted in the ICU wards of Golestan Hospital, Ahvaz, Iran, from September 2017 to March 2018. In this study, 52 patients with intracerebral hemorrhage were selected by convenient sampling (26 patients in each group) and were randomly assigned to two groups of melatonin and control. Sedation and pain management package was applied to both groups. Outcomes: Amount of the sedative and opioid drug; mechanical ventilation time; ICU staying time; Glasgow Coma Score; hemodynamic parameters.

Results There was no significant difference between them in terms of demographic characteristics. Cumulative doses of morphine and mechanical ventilation time were significant in two groups. $(P<0.05)$ The mean length of ICU staying was not significant. Glasgow Coma Score on the 6th day was significant in two groups $(P<0.05)$. Diastolic blood pressure was significant between groups $(P<0.001)$.

Conclusion This study presented that morphine consumption and mechanical ventilation time were significantly lower in the melatonin group than in the control. Also, rise in GCS in the melatonin group was faster in the melatonin group than in the control. The use of melatonin can be recommended for patients with ICH in the ICU for better outcomes.
\end{abstract}

Keywords Melatonin $\cdot$ Midazolam $\cdot$ Fentanyl $\cdot$ Morphine sulfate $\cdot$ Sedation $\cdot$ Intensive care unit $\cdot$ Critically ill

\section{Introduction}

ICH is most commonly caused by hypertension, arteriovenous malformations, or head trauma. Traumatic intracerebral hemorrhage (TICH) accounts for 9-27\% of strokes all inclusive. It is characterized by unfortunate results, with a high mortality rate of $30-50 \%$ and the neurological outcomes of patients who survive are also very poor [1]. On the other hand, evidence has shown that most patients who present with small ICHs can survive with good medical care $[2,3]$. This suggests that suitable medical involvements may improve the outcome of these patients $[4,5]$. The available

Fatemeh Javaherforooshzadeh

f_javaherforoosh@yahoo.com

1 Department of Anesthesia, Ahvaz Anesthesiology and Pain Research Center, Ahvaz Jundishapur University of Medical Sciences, Ahvaz, Iran data have shown that the early start of neuroprotective management might defend susceptible neurons and improve outcomes in hemorrhagic stroke [6]. Melatonin ( $N$-acetyl5 -methoxytryptamine) is a neurohormone that has attracted a great deal of interest as a therapeutic option for various neurological diseases because of its low toxicity, antioxidative, antiapoptotic, and anti-inflammatory properties. Also, it has some selective properties that are highly desired in the intensive care unit (IC) $[6,7]$. Melatonin was found to reduce inflammatory pain, probably by blocking the production of NO by inducible NO synthase and the signaling pathways of NO-cyclic GM. [7]. Melatonin is an effective free radical scavenger and an indirect antioxidant that has shown neuroprotective effects in both in vitro and in vivo ischemic-hypoxia models [8]. Decreased blood melatonin levels are associated with sepsis severity, delirium, and the severity of sleep alteration during critical illness.

The purpose of this study is to assess the effect of exogenous melatonin on the reduction in the need for sedative 
agents and the duration of mechanical ventilation in TICH patients in ICU.

\section{Methods}

\section{Trial design}

This is a double-blind, randomized controlled study with a parallel assignment of patients and ratio 1:1 between two groups. The local Ethics Committee of Ahvaz Jundishapur University of Medical Sciences, Ahvaz, Iran, approved all of the procedures of this study (IR.AJUMS.REC.1396.949). The RCT code of this study was: IRCT20190912044752N1. Written informed consent was collected from the families of the patients. After patients' neurological conditions improved, there were exactly well versed in the study, and their written consent was got both for their previously-collected records and further randomized treatments.

\section{Participants}

The study was conducted from September 2018 to May 2019 in patients admitted to the general ICU of Golestan Hospital, Ahwaz, Iran.

The inclusion criteria: age $\geq 18$ years, traumatic intracranial hemorrhage confirmed by computer tomography (CT) requiring surgery, initial GCS $4-8$, the average volume of ICH 30-35 cc and expected length longer than 2 days.

Exclusion criteria were: liver disease, renal insufficiency, pregnant women, patients with a brain tumor, and allergy to melatonin.

\section{Study settings}

The nurse to patient ratio was 1:2 during the morning, 1:3 during the evening and nightly, and intensivists were present in ICU at all times.

\section{Interventions}

Melatonin $3 \mathrm{mg}$ tablet (Webber Naturals® Canada; prolonged-release tablets) or control (placebo capsules filled by cornstarch powder (Beijing, China) was administered in the first $24 \mathrm{~h}$ at 9:00 p.m. and continued for all the days of hospitalization in ICU by NG tube. Patients were followed daily. According to the local guidelines, sedation started with intravenous midazolam $(0.25 \mathrm{mg} / \mathrm{kg} / \mathrm{h})$ and fentanyl $(2 \mu \mathrm{g} / \mathrm{kg} / \mathrm{h})$ that were discontinued as soon as possible. All tablets were directed by a nasogastric tube after shocking the tablet and mixing it with $20 \mathrm{~mL}$ of water, followed by another $20 \mathrm{~mL}$ to flush out the remainder. Opiates (morphine sulfate or methadone if the patient was addicted) were administered according to verbal rating scale $>3$ (VRS: verbal rating scales include the following five-point sets of descriptors which facilitate pain evaluation and treatment: "none", "mild", "moderate", "severe", "very severe" [9] or Behavioral Pain Scale $>6$ ( BPS, range 3-12, ) [10] and not as sedatives. Validated scales for pain and sedation/agitation monitoring (VRS or BPS) were used four times a day. Each morning, nurses blinded to melatonin treatment prescribed the daily therapy. In the presence of deeper-thandesired sedation levels, the prescribed drugs were decreased/ withdrawn. Conversely, according to clinical needs, an extra amount of drug was always allowed and recorded. Therapeutic decisions were made by blinded ICU physicians; neurological monitoring was performed by ICU nurses, also blinded to the group assignment.

\section{Outcomes}

Primary outcome was the amount of the most used sedative drug (sum of all midazolam and fentanyl doses administered, methadone, and morphine sulfate during the melatonin/placebo treatment).

Secondary outcomes were mechanical ventilation time; ICU staying time; Glasgow Coma Scale (GSC); hemodynamic parameters.

\section{Sample size}

Based on a predicted 33\% reduction from the patient's baseline, our sample size estimation indicated that 18 patients per group would give a power of 0.8 at an error 0.05 for detecting a $33 \%$ reduction in opioid consumption. Mean and standard deviations in opioid consumption were derived from Ismail et al. [11] and thus the total sample size was a prospective set of 40 patients. However, we increased our sample size to 60 patients to accommodate any withdrawal or missing data points.

\section{Randomization}

Treatment distribution was obtained through a computergenerated eight-patient block randomization procedure. After eligibility assessment at ICU admission, each patient received a sealed brown envelope containing the random melatonin or placebo treatment. It was then opened just evening of the first ICU day. Two physicians, according to Ethics Committee requirements, were aware of treatment allocations after the group assignment and monitored for possible side effects, without participating in clinical decisions about sedative administration or study treatment interruption. 


\section{Statistical analysis}

The statistical analysis was performed using a standard SPSS software package22 (Chicago, $\| \mathrm{IL}$ ). Data were expressed as mean values $\pm \mathrm{SD}$, percentages $(\%)$ and numbers $(n)$.

Independent samples $t$ test and paired-samples $t$ test were used to analyze the parametric data, and discrete (categorical) variables were analyzed using the $\chi^{2}$ test, with $P$ values $<0.05$ considered statistically significant.

\section{Results}

During the study period from September 2018 to May 2019 of Golestan Hospital, Ahwaz, Iran, in patients admitted in the general ICU, 80 patients were eligible to participate in the trial. After initial screening, 70 cases agreed to participate and their families provided informed consent. Among them, 18 patients did not satisfy inclusion criteria. Finally, 52 patients were enrolled in the study and were assigned into two groups of melatonin and placebo, 26 patients in each group (Fig. 1).

There was no statistically significant difference between the two groups in terms of demographic characteristics $(P>0.05)$ (Table 1).

\section{Primary outcomes}

The rate of cumulative dose of prescribed fentanyl and midazolam administration during admission in ICU was not statistically significant between the two groups. Cumulative methadone administration was not significant between groups, but cumulative doses of morphine that

Fig. 1 Consort flow diagram

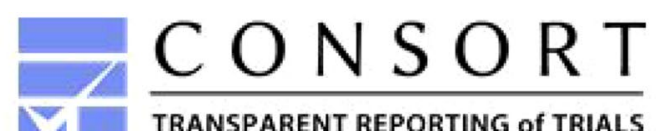

CONSORT 2010 Flow Diagram

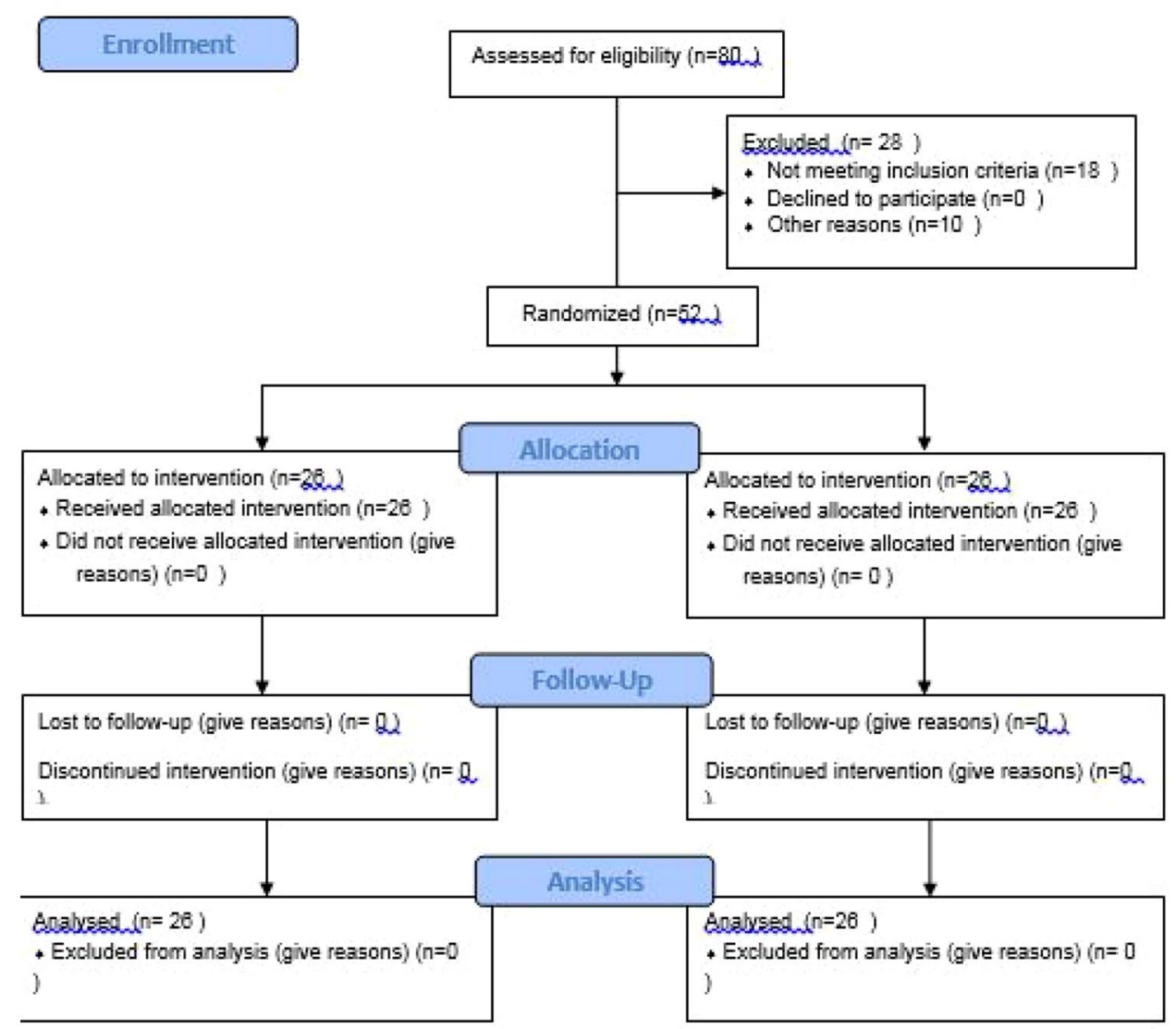


Table 1 Demographic and clinical data (mean $\pm \mathrm{SD}$ or count (\%)) of the study population

\begin{tabular}{llll}
\hline Variable & Melatonin group $(n=26)$ & Control group $(n=26)$ & $P$ value \\
\hline Age (year), mean \pm SD & $34.62 \pm 16.23$ & $36.85 \pm 17.47$ & $0.715^{\mathrm{a}}$ \\
Sex (male) $n(\%)$ & $18(69)$ & $19(73)$ & $0.45^{\mathrm{b}}$ \\
APACHE ||| & $7.45 \pm 4.24$ & $8.14 \pm 3.63$ & $0.35^{\mathrm{a}}$ \\
GCS 1 & $6.15 \pm 1.52$ & $6.25 \pm 1.04$ & $0.852^{\mathrm{a}}$ \\
\hline
\end{tabular}

$S D$ standard deviation, APACHE Acute Physiology and Chronic Health Evaluation, ICU intensive care unit

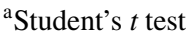

${ }^{\mathrm{b}} \chi^{2}$ test

were administered were significantly different between the groups $(P=0.037)$ (Table 2$)$.

\section{Secondary outcomes}

Mechanical ventilation time was significant between groups ( $7 \pm 3$ days in the melatonin group vs. $12 \pm 4$ in the control, $P=0.006$ ). The mean length of stay of the subjects in the ICU was calculated and compared in the melatonin and the control groups $(13.31 \pm 5.17$ days vs. $14.55 \pm 4.72$ days). The independent $t$ test showed that the length of stay was not significant between groups $(P=0.482)$. GCS at the beginning of hospitalization in the ICU (1st day) was not significant between the two groups, but GCS on the 6th day was $9.35 \pm 1.25$ in the melatonin group vs. $8.12 \pm 2.47$ in the control that was significant $(P=0.013)$ (Fig. 2, Table 3$)$.

GCS changes on day 6 compared to day 1 in the melatonin group $P<0.0001$. GCS changes on day 6 compared to day 1 in the control group $P=0.001$. GCS on the 6th day was $9.35 \pm 1.25$ in the melatonin group vs. $8.12 \pm 2.47$ in the control that was significant $(P=0.013)$.

Among the hemodynamic parameters, diastolic blood pressure was significant between groups $(76.54 \pm 5.158$ in the melatonin group vs. $83.35 \pm 4.511(P<0.001)$. Other parameters (systolic blood pressure, pulse rate, and $\mathrm{O} 2$ saturation) were not different between groups (Table 4).

\section{Discussion}

This study was a double-blind randomized controlled trial that was considered to evaluate the effect of melatonin on the need for sedative agents and duration of mechanical ventilation after TICH. Melatonin is a neurohormone created from the amino acid, tryptophan, and is mainly secreted by the pineal gland into the circulation [12]. The effectiveness of exogenous melatonin for the treatment of sleep disturbance [13], as antioxidant [14], antianxiety agent like benzodiazepine action has been established in many studies $[13,15$, 16]. Furthermore, different from the conventional sedative agents, this hormone does not deteriorate nighttime hypoxemia or ventilator responses [17-19]. Besides, it has been shown that melatonin can treat and avoid ICU delirium [20].

All of the patients with hemorrhagic stroke and loss of consciousness need to be mechanically ventilated. Although mechanical ventilation is often a life-giving treatment, its usage can result in unwanted side effects, including ventilator-associated lung injury, ventilator-associated pneumonia, sinusitis, gastrointestinal bleeding, and venous thromboembolism. [21, 22] It has been well recognized that the optimal use of the sedating agent may influence the duration of mechanical ventilation. Benzodiazepines and opiates are the most frequent drugs used for sedation and analgesia in the ICU. Both of these agents show some respiratory depressant effect, which can cause the duration of mechanical ventilation to be lengthy [23-25].

Melatonin is a neuroprotective agent with sedative, hypnotic, and analgesic properties and without any respiratory
Table 2 Comparison of cumulative dose of prescribed fentanyl, midazolam, methadone, and morphine sulfate between groups while in the ICU

\begin{tabular}{lccc}
\hline Drugs & Melatonin group (26) & Placebo group (26) & $P$ \\
\hline Fentanyl $(\mu \mathrm{g})$ & $820.00 \pm 144.625$ & $905.433 \pm 105.50$ & $0.354^{\mathrm{a}}$ \\
Midazolam $(\mathrm{mg})$ & $29.31 \pm 2.71$ & $37.45 \pm 3.25$ & $0.460^{\mathrm{a}}$ \\
Methadone $(\mathrm{mg})$ & $27.69 \pm 8.21$ & $42.25 \pm 5.72$ & $0.540^{\mathrm{a}}$ \\
Morphine sulfate $(\mathrm{mg})$ & $17 \pm 5.75$ & $30.82 \pm 6.98$ & $<0.0001^{\mathrm{a} *}$ \\
\hline
\end{tabular}

*Significant

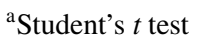


Fig. 2 Comparision of Glasgow Coma Scale between groups before and after intervension

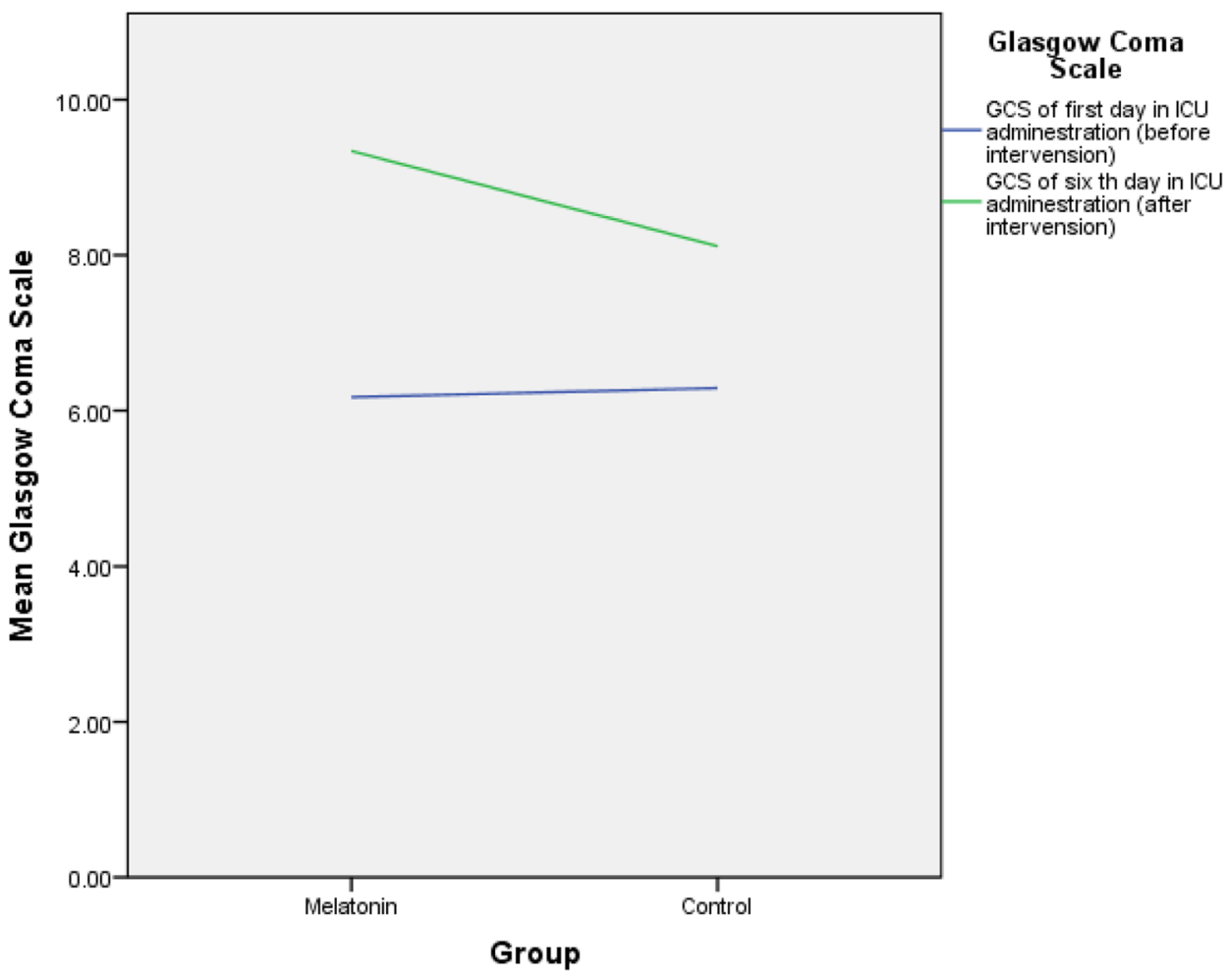

\begin{tabular}{lllr}
\hline Variable & $\begin{array}{l}\text { Melatonin group } \\
(n=26)\end{array}$ & Control group $(n=26)$ & $P$ value \\
\hline ICU staying time (days) & $13.31 \pm 5.17$ & $14.55 \pm 4.72$ & 0.482 \\
Mechanical ventilation time (days) & $7 \pm 3$ & $12 \pm 4$ & $0.006^{*}$ \\
Mortality rate $n$ (\%) & $2(7.69)$ & $8(30.77)$ & $<0.05^{*}$ \\
GCS 1 & $6.17 \pm 1.49$ & $6.28 \pm 1.09$ & 0.768 \\
GCS 6 & $9.31 \pm 1.09$ & $8.13 \pm 2.09$ & $0.013^{*}$ \\
\hline
\end{tabular}

*Significant at the level of 0.05

$S D$ standard deviation, ICU intensive care unit, GCS Glasgow Coma Scale

\begin{tabular}{lccr}
\hline Variable & Melatonin group $(n=26)$ & Control group $(n=26)$ & $P$ value \\
\hline SBP $(\mathrm{mmHg})$ & $127.69 \pm 12.182$ & $133.55 \pm 13.213$ & 0.209 \\
DBP $(\mathrm{mmHg})$ & $76.54 \pm 5.158$ & $83.35 \pm 4.511$ & $*<0.001$ \\
PR (rate/min) & $94.23 \pm 15.39$ & $94.15 \pm 16.007$ & 0.989 \\
$\mathrm{O}_{2}$ saturation $(\%)$ & $98.38 \pm 0.870$ & $97.75 \pm 2.149$ & 0.321 \\
\hline
\end{tabular}

*Significant at the level of 0.05

$S B P$ systolic blood pressure, $D B P$ diastolic blood pressure, $P R$ pulse rate
Table 4 Hemodynamic parameters comparison between groups
Table 3 Comparison of groups depressant effect which makes it a suitable adjuvant for sedation in the ICU $[17,25,26]$.

In Frisk et al.'s study, a reduced melatonin secretion was detected during mechanical ventilation [27]. Dessap et al. examined the association between melatonin levels of plasma and effective weaning from mechanical ventilation.
They determined that the lower plasma melatonin levels were linked with ineffective weaning [28].

In Dianatkhah et al.'s study, the duration of mechanical ventilation and length of ICU stay were shorter in patients who received melatonin in comparison with the control group, but this difference was statistically not significant 
for the length of ICU stay and marginally significant for the duration of mechanical ventilation $(P=0.065)$ [17]. In our study, as shown in Table 3, the duration of mechanical ventilation was shorter in patients given melatonin vs. the control group that was in line with the Frisk, Dessap, and Dianatkhah studies. On the other hand, the mean length of stay of the subjects in the ICU was not in line with Dianatkhah's study.

Our study included 52 patients. Results showed that lower doses of fentanyl and midazolam were used in the melatonin group, although this difference was not significant between the two groups. Also, the total dose of morphine sulfate in the melatonin group was significantly lower than the placebo. A study by Mistraletti et al. showed that administration of melatonin as an adjuvant in the ICU patients was associated with a reduction in the need for sedative agents, shortening the duration of mechanical ventilation, better neurological indicators, and cost reduction [29]. According to the literature, melatonin can be administered as an adjuvant with hypnotic properties and it may have shortened the number of hypnotic drugs and related side effects, including respiratory depression and delirium, which are both risk factors of lengthy weaning off from ventilator.

GCS is one of the determinants of neurological outcome in TICH patients. Many studies have shown the anti-inflammatory effect of melatonin that was similar to our results. Concerning the unusually high antioxidant effect of melatonin, it has been postulated that the administration of melatonin may be protective against ventilator injury [30,31].

As shown in Table 3, GCS was raised in the melatonin group in comparison with the control, which can be credited to the neuroprotective effect of melatonin. This finding was similar to that in Osier and coworker [32].

Li S and coworker presented that the melatonin attenuates early brain injury after subarachnoid hemorrhage by the JAK-STAT signaling pathway. One of the results of their study was melatonin treatment dramatically decreased neurological score and alleviated brain edema at $24 \mathrm{~h}$ after SAH $[33,34]$. In our study, GCS score on the 6th day was better in the melatonin vs. placebo group significantly. This finding was comparable with Li S's study.

\section{Limitations}

This study has some limitations. The first is that this study was a single-center with a guideline for sedative drugs that may be different from other ICU. Besides, trauma patients were compared with non-trauma patients. The third limitation was the lack of measurement of reactive oxygen species. The fourth limitation was we did not determine endogenous melatonin concentrations and circadian phase in our patients. Finally, our last limitation is the lack of information about the severity of head trauma, such as AIS and ICP. These are recommended for evaluation in future studies.

\section{Conclusion}

This study presented that morphine consumption and mechanical ventilation time were significantly lower in the melatonin group than the placebo group. Also rise in GCS in the melatonin group was faster in the melatonin group than in the placebo. Melatonin has sedative, analgesic, anticonvulsive, anti-inflammatory, and antioxidative properties that differentiate it as a new and an attractive drug in patients with mechanical ventilation. It is recommended to use melatonin for patients with ICH in the ICU for better tolerance of mechanical ventilation.

Acknowledgements We sincerely thank the patients who cooperated with us in this project and supported the research team. We want to thank the Clinical Research Development Unit, Golestan Hospital, Ahvaz Jundishapur University of Medical Sciences, and Ahvaz, Iran, for their cooperation.

Author contributions FS contributed to concept, study design, the definition of intellectual content, literature search, clinical studies, experimental studies, data acquisition, data analysis, and statistical analysis. FJ contributed to manuscript preparation, manuscript editing, and manuscript review. NN and KT participated in clinical and experimental studies. FK contributed to data collection.

Funding Financial support for this study was provided by Ahvaz Jundishapur University of Medical Sciences (Grant No: PAIN-9611 IR.AJUMS.REC.1396.949).

Data availability All data were retrieved from the institutional database and are available from the corresponding authors upon reasonable request.

\section{Compliance with ethical standards}

Conflict interest There is no conflict of interest to be declared.

Ethical approval This paper was part of a thesis. Ethics code: (IR.AJUMS.REC.1396.949). The RCT code of this study was: IRCT20190912044752N1.

Consent to participate Written informed consent was signed by the study patients.

Consent for publication The authors provide formal written consent for publication.

\section{References}

1. Qu J, Chen W, Hu R, Feng H. The injury and therapy of reactive oxygen species in intracerebral hemorrhage looking at mitochondria. Oxid Med Cell Longev. 2016;2016:9. 
2. Silva Y, Leira R, Tejada J, Lainez JM, Castillo J, Dávalos A, et al. Molecular signatures of vascular injury are associated with early growth of intracerebral hemorrhage. Stroke. 2005;36(1):86-91.

3. Sacco S, Marini C, Toni D, Olivieri L, Carolei A. Incidence and 10-year survival of intracerebral hemorrhage in a populationbased registry. Stroke. 2009;40(2):394-9.

4. Hemphill JC III, Greenberg SM, Anderson CS, Becker K, Bendok BR, Cushman M, et al. Guidelines for the management of spontaneous intracerebral hemorrhage: a guideline for healthcare professionals from the American Heart Association/American Stroke Association. Stroke. 2015;46(7):2032-60.

5. Hochart A, Cordonnier C. Emorragie cerebrali non traumatiche. EMC-Neurologia. 2017;17(4):1-11.

6. Andrabi SS, Parvez S, Tabassum H. Melatonin and ischemic stroke: mechanistic roles and action. Adv Pharmacolo Sci. 2015;2015:11.

7. Lekic T, Hartman R, Rojas H, Manaenko A, Chen W, Ayer R, et al. Protective effect of melatonin upon neuropathology, striatal function, and memory ability after intracerebral hemorrhage in rats. J Neurotrauma. 2010;27(3):627-37.

8. Rojas H, Lekic T, Chen W, Jadhav V, Titova E, Martin R, et al. The antioxidant effects of melatonin after intracerebral hemorrhage in rats. Cerebral Hemorrhage. Vienna: Springer; 2008. p. $19-21$.

9. Tsze DS, von Baeyer CL, Pahalyants V, Dayan PS. Validity and reliability of the verbal numerical rating scale for children aged 4 to 17 years with acute pain. Ann Emerg Med. 2018;71(6):691-702.

10. Hsiung N-H, Yang Y, Lee MS, Dalal K, Smith GD. Translation, adaptation, and validation of the behavioral pain scale and the critical-care pain observational tools in Taiwan. J Pain Res. 2016;9:661.

11. Ismail SA, Mowafi HA. Melatonin provides anxiolysis, enhances analgesia, decreases intraocular pressure, and promotes better operating conditions during cataract surgery under topical anesthesia. Anesth Analg. 2009;108(4):1146-51.

12. Dianatkhah M, Ghaeli P, Talasaz AH, Karimi A, Salehiomran A, Bina P, et al. Evaluating the potential effect of melatonin on the post-cardiac surgery sleep disorder. J Tehran Univ Heart Cent. 2015;10(3):122.

13. Grima NA, Rajaratnam SM, Mansfield D, Sletten TL, Spitz G, Ponsford JL. Efficacy of melatonin for sleep disturbance following traumatic brain injury: a randomised controlled trial. BMC Med. 2018;16(1):8.

14. Guo Z-N, Jin H, Sun H, Zhao Y, Liu J, Ma H, et al. Antioxidant melatonin: potential functions in improving cerebral autoregulation after subarachnoid hemorrhage. Front Physiol. 2018;9:1146.

15. Watson N, Diamandis T, Gonzales-Portillo C, Reyes S, Borlongan $\mathrm{CV}$. Melatonin as an antioxidant for stroke neuroprotection. Cell Transplant. 2016;25(5):883-91.

16. Barlow KM, Esser MJ, Veidt M, Boyd R. Melatonin as a treatment after traumatic brain injury: a systematic review and metaanalysis of the pre-clinical and clinical literature. J Neurotrauma. 2019;36(4):523-37.

17. Dianatkhah M, Najafi A, Sharifzadeh M, Ahmadi A, Sharifnia H, Mojtahedzadeh M, et al. Melatonin supplementation may improve the outcome of patients with hemorrhagic stroke in the intensive care unit. J Res Pharm Pract. 2017;6(3):173-7.

18. Maitra S, Baidya DK, Khanna P. Melatonin in perioperative medicine: current perspective. Saudi J Anaesth. 2013;7(3):315.
19. Halvani A, Mohsenpour F, Abadi EEF. Effect of melatonin on sleep quality of chronic obstructive pulmonary disease (COPD) patients. Eur Respir Soc. 2012;40:P3477.

20. de Jonghe A, Korevaar JC, van Munster BC, de Rooij SE. Effectiveness of melatonin treatment on circadian rhythm disturbances in dementia. Are there implications for delirium? A systematic review. Int J Geriatr Psychiatry. 2010;25(12):1201-8.

21. Ward D, Fulbrook P. Nursing strategies for effective weaning of the critically ill mechanically ventilated patient. Criti Care Nurs Clini. 2016;28(4):499-512.

22. Baid H. Patient safety: Identifying and managing complications of mechanical ventilation. Criti Care Nurs Clini. 2016;28(4):451-62.

23. Riker RR, Fraser GL. Adverse events associated with sedatives, analgesics, and other drugs that provide patient comfort in the intensive care unit. Pharmacother J Human Pharmacol Drug Ther. 2005;25(5P2):8S-18S.

24. Jarman A, Duke G, Reade M, Casamento A. The association between sedation practices and duration of mechanical ventilation in intensive care. Anaesth Intensive Care. 2013;41(3):311-5.

25. Javaherforooshzadeh F, Amirpour I, Janatmakan F, Soltanzadeh M. Comparison of effects of melatonin and gabapentin on post operative anxiety and pain in lumbar spine surgery: a randomized clinical trial. Anesth Pain Med. 2018;8(3):e68763.

26. Dianatkhah M, Najafi A, Sharifzadeh M, Ahmadi A, Sharifnia H, Mojtahedzadeh M, et al. Melatonin supplementation may improve the outcome of patients with hemorrhagic stroke in the intensive care unit. J Res Pharm Pract. 2017;6:173.

27. Frisk U, Olsson J, Nylén P, Hahn RG. Low melatonin excretion during mechanical ventilation in the intensive care unit. Clin Sci. 2004;107(1):47-53.

28. Dessap AM, Roche-Campo F, Launay J-M, Charles-Nelson A, Katsahian S, Brun-Buisson C, et al. Delirium and circadian rhythm of melatonin during weaning from mechanical ventilation: an ancillary study of a weaning trial. Chest. 2015;148(5):1231-41.

29. Mistraletti G, Umbrello M, Sabbatini G, Miori S, Taverna M, Cerri B, et al. Melatonin reduces the need for sedation in ICU patients: a randomized controlled trial. Minerva Anestesiol. 2015;81(12):1298-310.

30. Mistraletti G, Paroni R, Umbrello M, D’Amato L, Sabbatini G, Taverna M, et al. Melatonin pharmacological blood levels increase total antioxidant capacity in critically Ill patients. Int J Mol Sci. 2017;18(4):759.

31. Tordjman S, Chokron S, Delorme R, Charrier A, Bellissant E, Jaafari N, et al. Melatonin: pharmacology, functions and therapeutic benefits. Curr Neuropharmacol. 2017;15(3):434-43.

32. Osier N, McGreevy E, Pham L, Puccio A, Ren D, Conley YP, et al. Melatonin as a therapy for traumatic brain injury: a review of published evidence. Int J Mol Sci. 2018;19(5):1539.

33. Li S, Yang S, Sun B, Hang C. Melatonin attenuates early brain injury after subarachnoid hemorrhage by the JAK-STAT signaling pathway. Int J Clin Exp Pathol. 2019;12(3):909.

34. Javaherforooshzadeh F, Monajemzadeh SA, Soltanzadeh M, Janatmakan F, Salari A, Saeed H. A comparative study of the amount of bleeding and hemodynamic changes between dexmedetomidine infusion and remifentanil infusion for controlled hypotensive anesthesia in lumbar discopathy surgery: A double-blind, randomized, clinical trial. Anesthesiology and pain medicine. 2018;8(2). 